\title{
Charge-Carrier Dynamics in 2D Hybrid Metal-Halide Perovskites
}

\author{
Rebecca L. Milot, ${ }^{\dagger}$ Rebecca J. Sutton, ${ }^{\dagger}$ Giles E. Eperon, ${ }^{\dagger}$ Amir Abbas Haghighirad, ${ }^{\dagger}$ \\ Josue Martinez Hardigree, ${ }^{\dagger}$ Laura Miranda, ${ }^{\ddagger}$ Henry J. Snaith, ${ }^{\dagger}$ Michael B. Johnston, ${ }^{\dagger}$ and Laura M. Herz ${ }^{*}{ }^{\dagger}$ \\ ${ }^{\dagger}$ Department of Physics, University of Oxford, Clarendon Laboratory, Parks Road, Oxford, OX1 3PU, United Kingdom \\ \#Oxford Photovoltaics Ltd., Unit 6, Begbroke Science Park, Woodstock Road, Oxford, OX5 1PF, United Kingdom
}

Supporting Information

\begin{abstract}
Hybrid metal-halide perovskites are promising new materials for use in solar cells; however, their chemical stability in the presence of moisture remains a significant drawback. Quasi twodimensional (2D) perovskites that incorporate hydrophobic organic interlayers offer improved resistance to degradation by moisture, currently still at the cost of overall cell efficiency. To elucidate the factors affecting the optoelectronic properties of these materials, we have investigated the charge transport properties and crystallographic orientation of mixed methylammonium (MA)-phenylethylammonium (PEA) lead iodide thin films as a function of the MA-to-PEA ratio and, thus, the thickness of the

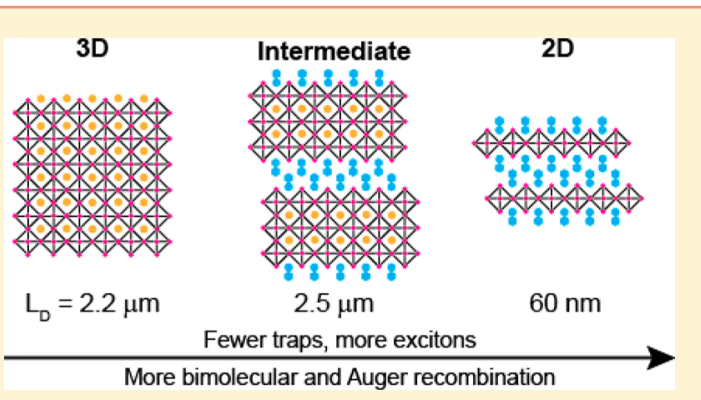
"encapsulated" MA lead-halide layers. We find that monomolecular charge-carrier recombination rates first decrease with increasing PEA fraction, most likely as a result of trap passivation, but then increase significantly as excitonic effects begin to dominate for thin confined layers. Bimolecular and Auger recombination rate constants are found to be sensitive to changes in electronic confinement, which alters the density of states for electronic transitions. We demonstrate that effective charge-carrier mobilities remain remarkably high (near $10 \mathrm{~cm}^{2} \mathrm{~V}^{-1} \mathrm{~s}^{-1}$ ) for intermediate PEA content and are enhanced for preferential orientation of the conducting lead iodide layers along the probing electric field. The trade-off between trap reduction, electronic confinement, and layer orientation leads to calculated charge-carrier diffusion lengths reaching a maximum of $2.5 \mu \mathrm{m}$ for intermediate PEA content (50\%).
\end{abstract}

KEYWORDS: Charge-carrier mobility, recombination, THz spectroscopy, GIWAXS, photovoltaics

$T^{k}$ he popularity of hybrid metal-halide perovskites for photovoltaic and other optoelectronic applications has risen steadily since their relatively recent debut in the field. ${ }^{1}$ This gaining interest is due largely to a combination of ease of synthesis and beneficial optoelectronic properties. Perovskite thin films can be synthesized via straightforward solutionprocessing and vapor-deposition techniques. ${ }^{2-4}$ Furthermore, they have high absorption coefficients across the visible spectrum, high charge-carrier mobilities, long carrier lifetimes, and long diffusion lengths, ${ }^{5,6}$ a valuable combination for thinfilm materials. After just a few years of development, efficiencies of state-of-the-art perovskite-based solar cells now exceed $22 \%$, approaching the Shockley-Queisser limit and rivalling existing inorganic semiconductor technologies. ${ }^{7}$

One challenge that hampers the widespread commercialization of perovskite-based solar cells is the chemical stability of the perovskite thin film. Although several factors including heat and UV light exposure influence perovskite stability, ${ }^{8-11}$ decomposition due to water exposure is one of the more pressing issues. In the prototypical hybrid perovskite methylammonium lead triiodide $\left(\mathrm{MAPbI}_{3}\right)$, the methylammonium cation is the main source of instability. ${ }^{10}$ Although many $\mathrm{Pb}^{2+}$ salts are only sparingly soluble in water, ${ }^{12}$ methylammonium is highly water-soluble. ${ }^{9}$ Furthermore, water is small enough to infiltrate the perovskite structure, where it can readily interact with methylammonium and form strong hydrogen bonds. ${ }^{13}$ At moderate exposure to ambient humidity, this interaction results reversibly in the formation of a hydrate, which, although stable, does not retain the optoelectronic properties of the water-free perovskite. ${ }^{13}$ Continued exposure to a humid environment exacerbates the problem and results in the complete, irreversible hydrolysis of methylammonium leading to the formation of $\mathrm{PbI}_{2} .{ }^{10}$

Attempts to solve the issue of water stability have focused on either encapsulating devices or improving the chemical stability of the perovskite layer itself. ${ }^{9,14-16}$ Because methylammonium aqueous solubility is the source of the problem, a promising strategy is to replace methylammonium with a less-watersoluble alternative. Replacing methylammonium with formamidinium has resulted in better thermal stability, but unfortunately, the moisture stability was not improved. ${ }^{9,17}$ One route to decrease the solubility of the organic cation would be to employ a larger hydrophobic functional group, but such ions typically exceed Goldschmidt's tolerance factor for the formation of perovskite structures. ${ }^{18}$ Stable perovskite-like structures are still possible with these cations, however, as the

Received: July 26, 2016

Revised: September 16, 2016

Published: September 30, 2016 
A

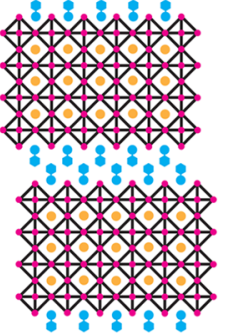

$40 \%$

\%PEA: $0 \%$

B
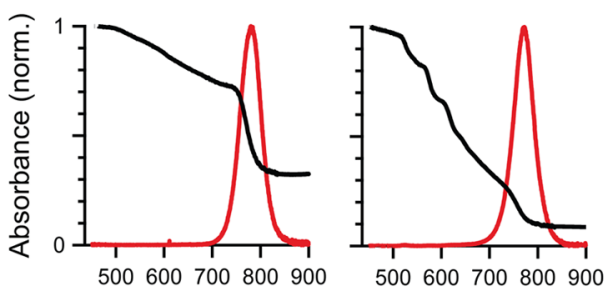

C
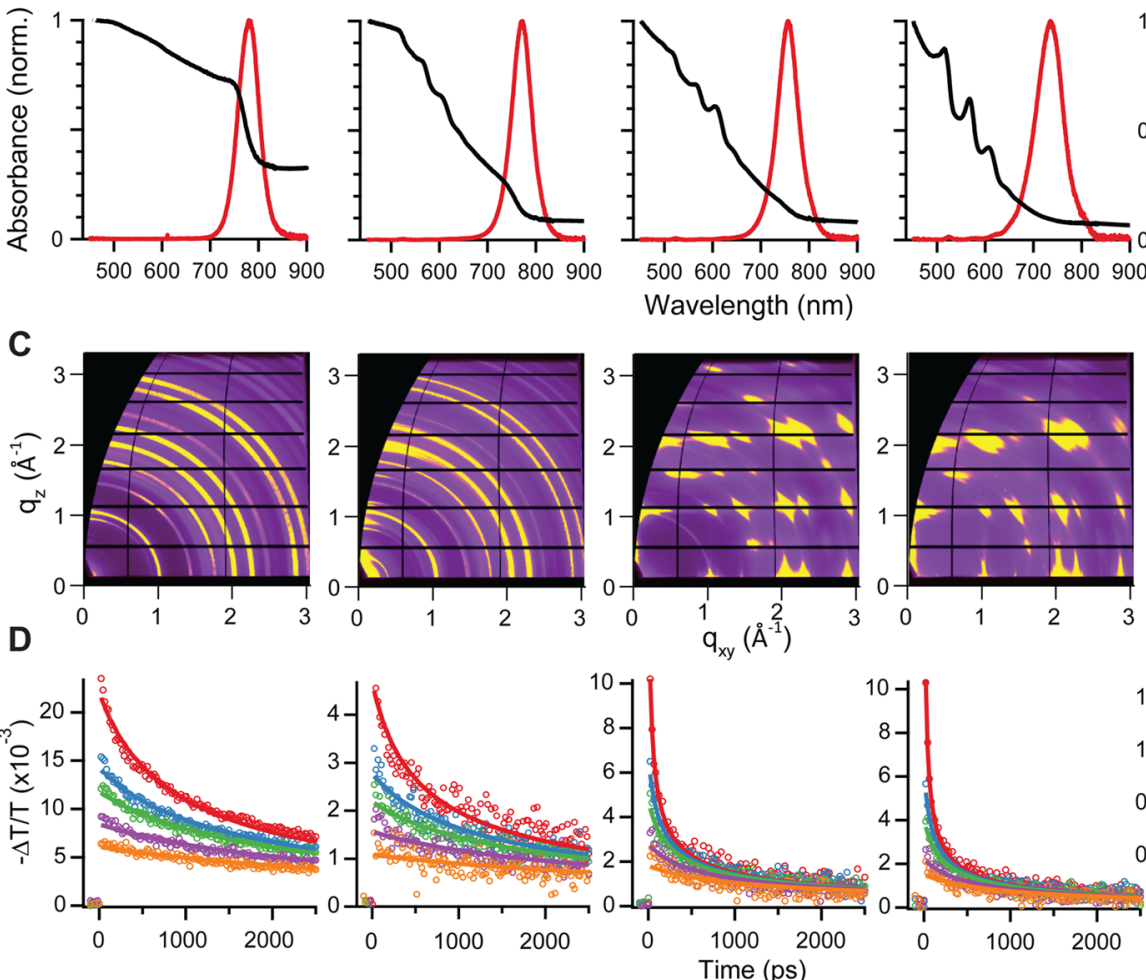

500600700800900
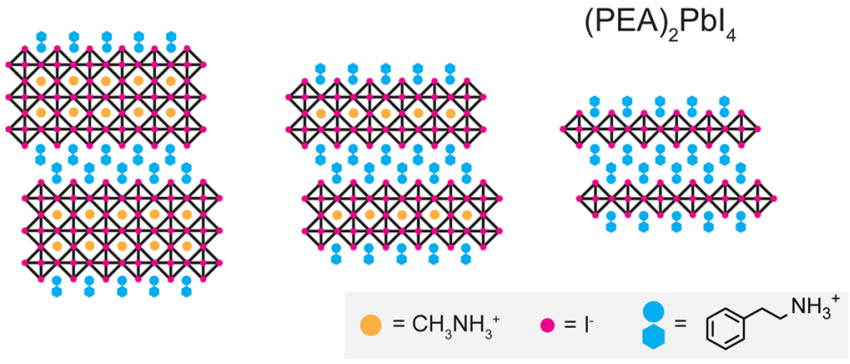

$67 \%$

$100 \%$
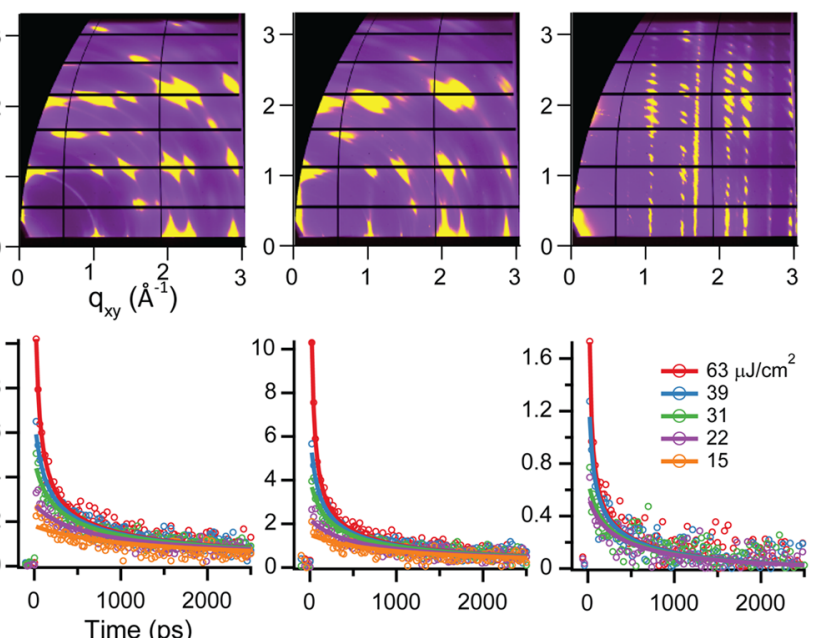

Figure 1. (A) Schematic crystal structures of $\mathrm{MAPbI}_{3},(\mathrm{PEA})_{2} \mathrm{PbI}_{4}$, and intermediate mixed MA-PEA 2D perovskites. Note that the $\mathrm{Pb}^{2+}$ ions are obscured by $\mathrm{I}^{-}$ions in this particular representation. Additionally, the \%PEA labels indicate the mole percentage of PEA as a fraction of the total organic cation composition. The labels for \%PEA also apply to parts B-D. (B) Normalized absorbance (left axis) and photoluminescence (right axis) spectra of the thin films investigated. We note that the nonabsorbing region does not show zero absorption due to scattering. (C) GIWAXS patterns in the scattering vector $\mathbf{q}$, which are representative of the top $100 \mathrm{~nm}$ depth of the film's surface for each composition. (D) Charge carrier dynamics measured with optical pump-THz probe spectroscopy. The samples were photoexcited at $400 \mathrm{~nm}$ with fluences ranging between $15-63$ $\mu \mathrm{J} / \mathrm{cm}^{2}$. The open circles are experimental data, and the solid lines are fits to eq 1 .

larger cations can force a two-dimensional layered structure, as shown in Figure 1A. In the most common arrangement, a layer of corner-sharing metal halide octahedra cut along the $<100>$ direction with respect to the original perovskite structure is separated by a nanometer-scale bilayer of monovalent, organic cations. ${ }^{19}$ These structures are highly tunable, and adding methylammonium in stoichiometric mixtures results in structures with varying thicknesses of a "perovskite" metal halide layer between the bilayers of the larger organic cations (Figure 1A). ${ }^{20}$ In all cases, the resulting structures can be considered a type of nanoscale encapsulation in which the metal-halide layers are protected from water infiltration by hydrophobic organic layers.

The use of two-dimensional (2D) perovskite materials in solar cells was initially explored in two recent reports: one study used butylammonium ${ }^{21}$ as the hydrophobic cation, and the other used 2-phenylethylammonium (PEA). ${ }^{22}$ In both instances, cells made with $2 \mathrm{D}$ perovskites were stable in air for at least two months, and their three-dimensional (3D) counterparts showed signs of degradation after just a few days. Although the stability was dramatically improved, the maximum recorded power conversion efficiencies (PCEs) of these cells did not exceed $8 \%$. It is unclear from these two initial solar cell studies whether the low efficiencies are the result of unoptimized processing or fundamental problems with charge transport in $2 \mathrm{D}$ perovskites, although a few other studies have suggested that the $2 \mathrm{D}$ nature of these perovskites should not limit device performance, ${ }^{23,24}$ and others have reported improved device efficiencies of both photovoltaic and lightemitting devices with synthetic manipulation of the 2D materials. $^{25-27}$ Furthermore, one of the original studies also compared the performance of solar cells based on 2D 
perovskites with varying lead iodide layer thickness and observed a maximum PCE for a film containing 50\% butylammonium, suggesting a nontrivial relationship between efficiency and the physical and chemical properties of the film. ${ }^{21}$

One important consideration for charge transport in $2 \mathrm{D}$ perovskites is the effect of quantum confinement. Due to an electronic energy level offset between the semiconducting lead iodide layers and the insulating organic components and a mismatch in their dielectric constants $\left(\varepsilon_{\text {organic }} \approx 2\right.$ and $\varepsilon_{\text {perovskite }}$ $\approx 6),{ }^{28}$ a quantum well forms within the inorganic layer. ${ }^{9,28}-30$ become confined to the inorganic layer, although DFT calculations suggest that the inorganic layers can be very weakly interacting. ${ }^{29}$ As a result, the photoconductivity within the lead iodide layers is much greater than the photoconductivity across the layers. ${ }^{31,32}$ Unless the films are perfectly oriented such that the lead iodide layers are perpendicular to the substrate, this anisotropy could be a significant hindrance to efficient charge transport. Interestingly, improvements in solar cell performance have been attributed both to randomizing the orientation and to tuning the orientation for favorable charge transport. $^{21,25,26}$ Another consideration that arises from the energetic and dielectric mismatch is that the exciton binding energy increases with decreasing lead iodide layer thickness and can be as high as $300 \mathrm{meV} .^{28,33-35}$ Because excitonic effects tend to enhance charge-carrier recombination rates, chargecarrier diffusion tends to be curtailed, as in the case of organic semiconductors, where similarly high exciton binding energies typically limit exciton diffusion lengths to well below $100 \mathrm{~nm} .{ }^{36}$ Therefore, the presence of excitons at room temperature could present a problem for the planar device structures favored for perovskite solar cells, which require diffusion lengths of several hundred nanometers. ${ }^{37}$

This study explores the fundamental charge transport in $2 \mathrm{D}$ perovskites by investigating the effects of changing the MA-toPEA ratio in $\mathrm{MA}_{n-1} \mathrm{PEA}_{2} \mathrm{~Pb}_{n} \mathrm{I}_{3 n+1}$ thin films on the charge carrier mobility, recombination dynamics, and diffusion length and examining how these properties relate to the orientation within the film. We find that the optoelectronic properties of these materials result from a balancing between decreased trapping, preferential orientation of the crystallites, and increasing excitonic character that results in optimum properties at 50\% PEA. Our study hence explains previously observed trends with PEA fraction in the performance of photovoltaic cells incorporating such $2 \mathrm{D}$ perovskites.

Thin films of varying \%PEA were prepared by spin-coating a $1 \mathrm{M}$ precursor solution in $\mathrm{N}, \mathrm{N}$-dimethylformamide (DMF) onto z-cut quartz substrates for optical and charge transport measurements and onto glass substrates for X-ray characterization. All samples were annealed at $70{ }^{\circ} \mathrm{C}$ for $10 \mathrm{~min}$; we note that this process was optimized for the mixed MA-PEA perovskites and is not optimal for $\mathrm{MAPbI}_{3}$. Full experimental details may be found in the Supporting Information.

A change in composition through the series was confirmed by analysis of their optical absorbance (UV-vis) and photoluminescence (PL) spectra (Figure 1B). The 0\% PEA sample $\left(\mathrm{MAPbI}_{3}\right)$ displays a band edge centered near $776 \mathrm{~nm}$ and corresponding PL peak at $780 \mathrm{~nm}$. The nonzero value of the absorbance at energies below the absorption onset is consistent with a large amount of scattering in this particular sample, as the sample synthesis method, which was optimized for $2 \mathrm{D}$ perovskites, produced a rather rough film morphology.
The band edge position and PL maximum lie to the lower energy edge of the range of reported literature values, suggesting that the sample is highly crystalline and should therefore possess good short-range charge-carrier properties. $^{38,39}$ The $100 \%$ PEA sample, which is visually very smooth and does not exhibit much scattering below the absorption onset, displays a strong absorption peak at $514 \mathrm{~nm}$ with the accompanying PL peak at $526 \mathrm{~nm}$, which have previously been attributed to excitonic absorption and emission, respectively. ${ }^{28,40}$ The films composed of mixtures of MA and PEA, conversely, all have broad spectra that display several absorption peaks between 500 and $700 \mathrm{~nm}$, suggesting the presence of domains containing varying PEA-MA composition, resulting in a range of different lead iodide layer thicknesses. However, the higher energy excitonic peaks, which correspond to species with thinner lead iodide layers (higher concentrations of PEA), are known to be more intense and sharper than the lower energy peaks. ${ }^{20,22}$ Therefore, regions of higher PEA concentration are likely to contribute disproportionately to the absorption while only being present in trace amounts. 22,35

The PL spectra further confirm the presence of lowerdimensionality minority species in the mixed MA-PEA films. To test sample uniformity, PL spectra were taken following photoexcitation from the front surface and also from the substrate-contacted back surface of the thin films (Figure S1). In both cases, the blue shift of the PL maxima with increased \%PEA indicates increased confinement as the thickness of the lead iodide layer decreases. The comparatively large blue shift from $67 \%$ PEA to $100 \%$ PEA arises because the compositional change is accompanied by a halving of nominal layer thickness, ${ }^{28}$ and the confinement energy of an infinite quantum well is proportional to $1 / d^{2}$, where $d$ is the well thickness. ${ }^{41}$ When the samples are excited from the front surface, a single peak is observed for all of the samples, but with back excitation, additional and higher energy peaks are observed. The presence of these additional peaks for back excitation indicates that the regions of higher \%PEA, which were also detected in the absorption spectra, are likely concentrated at the back surface of the film. Such slight compositional gradients in the material may, for example, be caused by differences in solubility of the PEAI and MAI precursors in the solvent employed (DMF). The low intensities of these peaks further suggest that higher $\%$ PEA species represent a minority fraction of the overall composition, and thus, the samples are relatively uniform on the whole. This result is also supported by XRD (Figure S7) data, in which the presence of very-low-intensity peaks at angles indicative of $100 \%$ PEA in all of the mixed PEA-MA films suggests only a minor contribution from higher \%PEA species.

Previous studies have indicated that the layers in $2 \mathrm{D}$ perovskites can align either parallel or perpendicular to the surface of the substrate upon which they are grown. ${ }^{20,21,25}$ To determine any preferential orientation in our films, we performed grazing-incidence wide-angle X-ray scattering (GIWAXS) measurements. Figure 1C shows GIWAXS patterns in the scattering vector $\mathbf{q}$, which are representative of the top $100 \mathrm{~nm}$ of the film surface for each composition. Overall, we find an increase in preferential orientation with increasing \% PEA. With no PEA in the film $\left(\mathrm{MAPbI}_{3}\right)$ we see DebyeScherrer rings, which are characteristic of a polycrystalline, powder-like film. A similar pattern of Debye-Scherrer rings is observed for the $40 \%$ PEA film, suggesting that it is also polycrystalline and powder-like. As the proportion of PEA in 
the film is increased beyond $40 \%$, we observe intense broad spots on the Debye-Scherrer rings, indicative of preferential orientation of crystallites in the film. For the 100\% PEA sample, these spots form lines at constant $\mathbf{q}_{x y}$ or at constant $\mathbf{q}_{z}$ revealing layering within the film both in-plane and out-ofplane with respect to the substrate. Further investigation of the GIWAXS data for the 100\% PEA sample (see the Supporting Information) confirms that the layers are oriented such that the lead iodide planes are parallel with the substrate, i.e., with the $c$ axis perpendicular to the substrate, with some stacking faults deeper within the film. We see little change with depth in the top 300-400 $\mathrm{nm}$ of all the films, indicating uniform composition throughout the top half to third of the films. Some strain is evident on the top surface of the film, although this is convoluted in the sample volume. A detailed analysis of the GIWAXS data (attenuation lengths, structure determination, and changes with depth) is provided in the Supporting Information.

To probe the optoelectronic properties of these materials, we proceed by determining the effective charge-carrier mobility in the thin films (Figure 2) using optical pump-THz probe

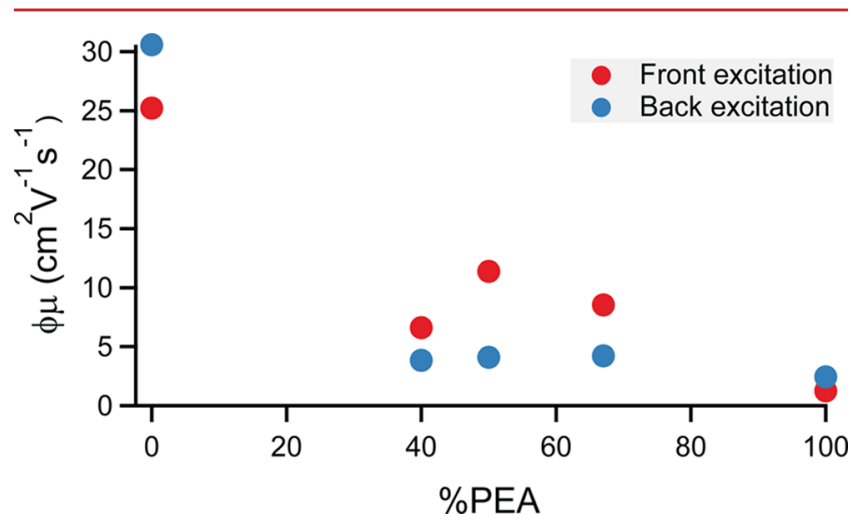

Figure 2. Effective charge-carrier mobility $(\varphi \mu)$ determined from optical pump-THz probe measurements. Both values were obtained by exciting the samples either from the front side (with the perovskite film first, red markers) or the substrate side first (blue markers). Film thicknesses were all greater than $550 \mathrm{~nm}$ (see the Suppporting Information), which is significantly larger that the optical absorption depth at the $400 \mathrm{~nm}$ excitation wavelength (less than $100 \mathrm{~nm}$ for all films). The error for all mobility values is estimated to be $6 \%$.

spectroscopy (OPTP). The samples were excited at $400 \mathrm{~nm}$ at normal incidence, for which the probing $\mathrm{THz}$ electric field vector lies in the plane of the film. For the oriented samples (50-100\% PEA), this setup thus probes the mobility for charge motion along the lead-iodide layers. We note that because charge extraction in a thin-film photovoltaic cell occurs generally along the direction perpendicular to the film, optimized cells would require $2 \mathrm{D}$ layers to be oriented perpendicular to the substrate. Hence, our experiments tentatively explore the charge-carrier mobility for such optimized geometry, in which charge conduction occurs predominantly along the $2 \mathrm{D}$ lead iodide planes. Following photoexcitation, the fractional change in $\mathrm{THz}$ amplitude $(\Delta T /$ $T$ ) was monitored as a function of the time delay between the optical pump pulse and $\mathrm{THz}$ probe pulse, yielding traces shown in Figure 1D. Here, the $\Delta T / T$ signal can be converted into the photoinduced conductivity, which is proportional to the product of the carrier density and the mobility $\mu$ (see the Supporting Information for details). However, because these experiments do not contain information on the fraction $\varphi$ of absorbed photons that is converted into free charge-carrier pairs, only an effective mobility, $\varphi \mu$, can be determined, in which $\varphi$ is the photon-to-charge branching ratio. In 3D perovskites for which the contribution of excitonic effects at room temperature is negligible, ${ }^{6} \varphi=1$, and the effective chargecarrier mobility equals the actual mobility. However, due to the enhancement of the exciton binding energy in 2D perovskites, we can no longer generally assume that $\varphi=1$; therefore, we report effective charge-carrier mobilities $(\varphi \mu)$.

Because the PL measurements showed differences between the materials near the front surface and the bulk (near the substrate), we determined the mobility exciting both the front (contacted with air) and the back (contacted with the quartz substrate) surfaces of the thin films. Because the penetration depth at $400 \mathrm{~nm}$ excitation is less than $100 \mathrm{~nm}$ for all of the films investigated, the measured $\Delta T / T$ values represent weighted averages, which favor contributions from either the front or the back surfaces. As shown in Figure 2, the effective charge-carrier mobility for the $2 \mathrm{D}$ films is lower when exciting from the back, which is consistent with the presence of some excess of (PEA) $)_{2} \mathrm{PbI}_{4}(100 \%$ PEA) and other lower dimensionality perovskites at the back surface of all of the films. We will therefore focus our attention on charge-carrier mobilities and dynamics obtained when exciting from the front surface of the film as they are more representative of the desired single-component materials and are therefore less-influenced by minority species.

We find that for front excitation the effective charge-carrier mobility generally decreases with increasing PEA content from $25 \mathrm{~cm}^{2} \mathrm{~V}^{-1} \mathrm{~s}^{-1}$ for $\mathrm{MAPbI}_{3}$ to $1 \mathrm{~cm}^{2} \mathrm{~V}^{-1} \mathrm{~s}^{-1}$ for (PEA) $)_{2} \mathrm{PbI}_{4}$. We attribute this to an increase in charge-carrier confinement and the concomitant increase in exciton binding energy as the $\%$ PEA increases. $^{28}$ While the charge-carrier mobility of $(\mathrm{PEA})_{2} \mathrm{PbI}_{4}$ is low in comparison to that of perovskites typically used in solar cells, the mobilities of all of the MA-PEA mixtures, which are between 6 and $11 \mathrm{~cm}^{2} \mathrm{~V}^{-1} \mathrm{~s}^{-1}$, are within the range of values reported for perovskite films capable of producing efficient devices. ${ }^{5}$ In addition to excitonic effects, the charge-carrier mobilities of the mixed MA-PEA samples are also influenced by layer alignment and disorder effects. Compared to the other mixed samples, the $40 \%$ PEA film had the lowest mobility $\left(6 \mathrm{~cm}^{2} \mathrm{~V}^{-1} \mathrm{~s}^{-1}\right)$, most likely due to its lack of preferential layer alignment with respect to the $\mathrm{THz}$ electric field probe. As a result of the random orientation, the chargecarrier mobility value therefore represents an average in the directions along and perpendicular to the layers. The 50\% PEA sample benefits from improved orientation and thus exhibits the highest charge-carrier mobility value of all of the $2 \mathrm{D}$ thin films $\left(11 \mathrm{~cm}^{2} \mathrm{~V}^{-1} \mathrm{~s}^{-1}\right)$. For PEA fractions higher than $50 \%$, the effects of increasing exciton binding energy outweigh the benefits of improved orientation and the effective mobility decreases significantly in accordance with a reduced value of $\varphi$.

To better understand the charge transport mechanism in these materials, we unraveled the charge carrier dynamics using OPTP measurements in combination with time-resolved photoluminescence. As can be seen qualitatively in Figure $1 \mathrm{D}$, the overall charge-carrier recombination rate generally increases with increasing PEA content, although further analysis reveals that the dynamics are more subtle. To separate competing charge-carrier recombination mechanisms, we fitted the decay traces in Figure 1D to the following charge-carrier recombination rate equation: ${ }^{5,6}$ 
A

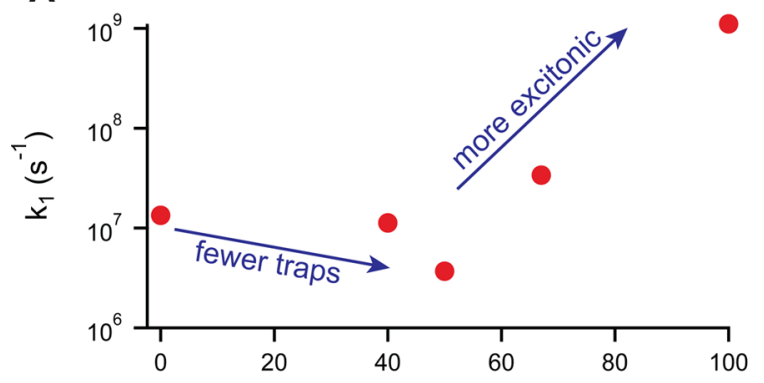

C

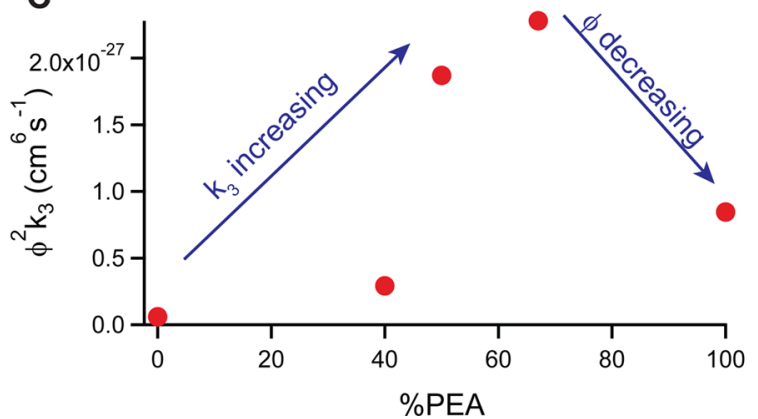

B

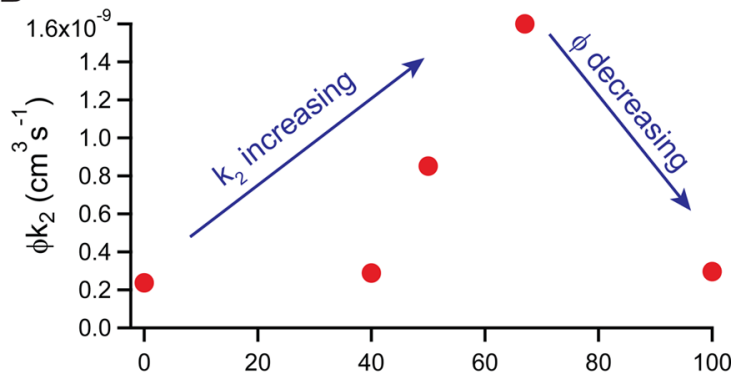

D

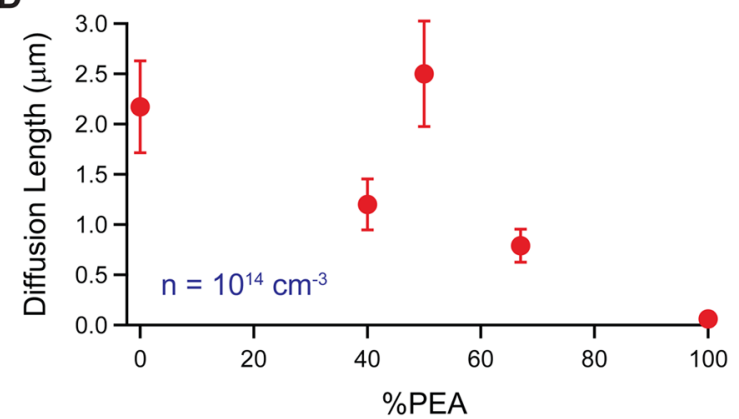

Figure 3. Recombination rate constants and diffusion length. (A) Monomolecular recombination rate $\left(k_{1}\right)$ determined from time-resolved PL measurements shown in Figure S2. The error in the measured values is estimated to be $20 \%$. (B) Effective bimolecular rate constant $\left(\varphi k_{2}\right)$ extracted from fits to OPTP traces shown in Figure 1D. The error is estimated to be $10 \%$. (C) Effective Auger rate constant $\left(\varphi^{2} k_{3}\right)$ extracted from fits to OPTP traces shown in Figure 1D. The error is estimated to be $16 \%$. (D) Charge carrier diffusion length at a charge carrier concentration of $10^{14}$ $\mathrm{cm}^{-3}$.

$$
\frac{\mathrm{d} n}{\mathrm{~d} t}=-k_{3} n^{3}-k_{2} n^{2}-k_{1} n
$$

where $n$ is the charge-carrier density, $k_{1}$ is the monomolecular recombination rate, $k_{2}$ is the bimolecular recombination rate constant, and $k_{3}$ is the Auger rate constant. Values of $k_{1}$ were obtained from time-resolved PL measurements, which allow for the determination of the total (radiative and nonradiative) monomolecular recombination rate. Effective values for the bimolecular and Auger recombination constants, $\varphi k_{2}$ and $\varphi^{2} k_{3}$, were obtained by global fits to the fluence-dependent $\mathrm{THz}$ data (see the Supporting Information for details of the fitting procedure).

Figure 3 shows the charge-carrier recombination rate constants obtained from both PL and OPTP measurements. For $\mathrm{MAPbI}_{3}$, the monomolecular rate (Figure $3 \mathrm{~A}$ ) is $1.3 \times 10^{7}$ $\mathrm{s}^{-1}$, which is consistent with previous reports. ${ }^{5}$ With increasing PEA content, $k_{1}$ first decreases by almost an order of magnitude to $3.7 \times 10^{6} \mathrm{~s}^{-1}$ for 50\% PEA and then increases by nearly 3 orders of magnitude to $1.1 \times 10^{9} \mathrm{~s}^{-1}$ for $(\mathrm{PEA})_{2} \mathrm{PbI}_{4}$. In $3 \mathrm{D}$ perovskites, the monomolecular recombination is dominated by charge-carrier trapping at impurities, and decreasing the concentration or effectiveness of trap states (for example, by altering the surface chemistry or decreasing the temperature) leads to considerable decreases in the monomolecular recombination rate. ${ }^{42-45}$ We propose that a similar passivation mechanism is operating for the $2 \mathrm{D}$ perovskite samples examined here. At small to moderate concentrations, PEA could effectively passivate the surface of the perovskite layers or grain boundaries, improving film quality and decreasing the monomolecular recombination rate. However, as the \% PEA increases, the exciton binding energy increases due to enhanced quantum confinement and the probability of exciton formation also increases, ${ }^{28}$ which adds an additional monomolecular recombination pathway. Due to the increase in binding energy with higher \%PEA, it is likely that this pathway is radiative, which could potentially be a benefit for light emission and lasing applications. Above 50\% PEA content, monomolecular exciton recombination hence counteracts the reduction in $k_{1}$ achieved through passivation, and we find that $k_{1}$ increases substantially (Figure 3A).

Interestingly, the bimolecular and Auger recombination rate constants (Figure 3B,C) both exhibit similar trends, increasing until 67\% PEA and then decreasing for the 100\% PEA film. We attribute the initial increase in $\varphi k_{2}$ with PEA content to the increase in the electronic density of states near the band edge that is generally encountered with increased quantum confinement. ${ }^{41}$ While for an idealized three-dimensional bulk semiconductor with parabolic bands, the joint density of states for photoinduced transitions rises from the band edge with the square-root of the photon energy, for a two-dimensional semiconductor, a sharp onset to a constant density of states is expected. Such effects are generally exploited to lower the threshold for lasing by lowering the dimensionality of the semiconductor, which enhances the radiative electron-hole recombination through a higher density of states near the bandedge states into which carriers relax following injection. The observed initial increase in $\varphi k_{2}$ with increasing PEA content nicely confirms these expectations and suggests that addition of a fraction of PEA may also be an interesting strategy to pursue for lowering of thresholds for amplified spontaneous emission in hybrid perovskite laser applications and also for improving the quantum efficiency of perovskite LEDs. ${ }^{46}$ The fully 2D material (100\% PEA) deviates from this trend, exhibiting a value of $\varphi k_{2}$, which is significantly lower than that observed for the material with 67\% PEA. We attribute this decrease at high PEA content again to the increased propensity for exciton formation, which leads to an accompanying decrease in the 
photon-to-charge branching ratio $\varphi$ that counteracts the rise in $k_{2}$ in the measured quantity $\varphi k_{2}$.

A similar analysis can be applied to the trends observed for the effective Auger rate constant $\varphi^{2} k_{3}$. Until 67\% PEA fraction, $\varphi^{2} k_{3}$ increases due to increases in $k_{3}$ but decreases for the $100 \%$ PEA film due to a decrease in $\varphi$. The Auger rate in semiconductors can be influenced by many factors including the nature of the band structure, the presence of phonons, and the concentration of impurities. ${ }^{4-50}$ In quantum confined systems, additional factors may affect Auger rate constants. The larger Coulombic interactions resulting from increased confinement may enhance the Auger rate, and the decreased availability of states satisfying energy conservation may decrease the rate. ${ }^{51}$ In semiconductor quantum dots, the Auger recombination rate constant was found to increase with decreasing particle diameter and increasing quantum confinement, suggesting the enhancement of Coulomb interactions to be the dominant factor. ${ }^{51,52}$ Similar trends are predicted for semiconductor quantum wells with varying well thickness. ${ }^{50,53}$ We therefore propose in analogy that the underlying trend of increasing Auger rate constant we observe with increasing \% PEA fraction results from increased quantum confinement, similar to what has been observed for inorganic semiconductor nanostructures.

On the basis of our analysis of charge-carrier recombination and mobility values, we are able to evaluate the charge-carrier diffusion length for each material, which serves as an important indicator for thin-film photovoltaic performance. Efficient charge-carrier collection at electrodes requires a charge-carrier diffusion length well in excess of the film thickness and optical absorption depths at harvested solar photon energies. Hence, we proceed to calculate charge-carrier diffusion lengths according to the following equation:

$$
L_{D}(n)=\sqrt{\frac{D}{R_{\text {total }}(n)}}
$$

where $R_{\text {total }}=-\frac{1}{n} \frac{\mathrm{d} n}{\mathrm{~d} t}=n^{2} k_{3}+n k_{2}+k_{1}$ and $D=\frac{\mu k_{\mathrm{B}} T}{e}$. A charge-carrier density of $10^{14} \mathrm{~cm}^{-3}$ was used to represent typical conditions under low-level solar illumination, for which recombination is typically monomolecular in hybrid perovskite materials. ${ }^{5}$ Therefore, the evaluated charge-carrier diffusion lengths displayed in Figure 3D derive from the combined trends observed in the charge-carrier mobility (Figure 2) and monomolecular recombination rate $k_{1}$ (Figure $3 \mathrm{~A}$ ) with changing PEA fraction. The underlying decline in the chargecarrier diffusion length with increasing \%PEA (from $2.2 \mu \mathrm{m}$ for $\mathrm{MAPbI}_{3}$ to $60 \mathrm{~nm}$ for $(\mathrm{PEA})_{2} \mathrm{PbI}_{4}$ ) can then be understood in terms of the declining trend in charge-carrier mobility and the increased excitonic effects with PEA fraction. For the film with intermediate amount (50\%) of PEA, however, a maximum in charge-carrier diffusion length is reached, at a remarkably good value of $2.5 \mu \mathrm{m}$. For this intermediate regime, excitonic effects are still relatively subtle, monomolecular recombination is lowest because of effective trap passivation, and the chargecarrier mobility is relatively high as a result of enhanced ordering of layers in the direction of the electric-field probe. Therefore, by unraveling the complex factors contributing to the overall charge-diffusion lengths, we are able to explain the counterintuitive result that the addition of an insulating material leads to better performance in solar cells. ${ }^{22}$ For the materials investigated here, the lowering of trap-related recombination rates dominates over the slighter lowering of charge-carrier mobility with addition of up to 50\% PEA; hence, the charge-carrier diffusion length is enhanced. We also note that because $k_{1}$ is determined largely by the concentration of defects (for all but the strictly 2D materials), the overall performance may be altered through different processing techniques. More recent studies support this conclusion, as enhanced power conversion efficiencies have been obtained for solar cells based on $2 \mathrm{D}$ perovskites made through a variety of film preparation procedures. ${ }^{25,26,45,54}$

In conclusion, we have determined the optoelectronic properties of mixed MA-PEA 2D perovskites as a function of increasing \% PEA and found correlations with previously reported changes in the efficiency of solar cells based on similar materials. We show that the observed trends in optoelectronic properties result from a complex interplay of different factors. As the \%PEA increases, the thin films become more preferentially oriented until the crystallites are aligned parallel to the substrate along the $<001>$ direction for the $100 \%$ PEA film. Simultaneously, quantum confinement effects and exciton binding energy increase, which tend to decrease the chargecarrier mobility and increase bimolecular and Auger recombination rate constants. The monomolecular recombination rate is affected by two counteracting trends, decreasing first with increasing PEA content due to a trap passivation effect but then increasing as excitonic recombination is enhanced. We show that the trade-off between these counteracting trends leads to the intermediate 50\% PEA film exhibiting the highest chargecarrier diffusion length of $2.5 \mu \mathrm{m}$, which is higher than many reported values for even the three-dimensional hybrid perovskites. Our study demonstrates that 2D perovskites with optimized alignment of lead iodide transport layers can exhibit an ideal combination of reduced trap-related recombination and decent charge-carrier mobilities, yielding water-resistant materials for highly efficient photovoltaic cells.

\section{ASSOCIATED CONTENT}

\section{Supporting Information}

The Supporting Information is available free of charge on the ACS Publications website at DOI: 10.1021/acs.nanolett.6b03114.

Additional experimental details, $\mathrm{THz}$ data analysis procedures, additional steady-state PL spectra, timedependent PL spectra, and GIWAXS analysis. Tables showing film thicknesses, $d$-spacings for [00l] lattice planes from simulated and measured structures, lattice parameters for simulated and measured $(\mathrm{PEA})_{2} \mathrm{PbI}_{4}$, and approximate densities calculated from experimental and literature values. Figures showing a comparison of steadystate photoluminescence spectra, time-dependent photoluminescence, GIWAXS patterns in $\mathbf{q}_{x y}-\mathbf{q}_{z}$ for (PEA) $)_{2} \mathrm{PbI}_{4}, \mathrm{XRD}$ patterns, (PEA) ${ }_{2} \mathrm{PbI}_{4}$ in the eclipsed and staggered structures, attenuation lengths for each composition, powder XRD patterns $\left(\mathrm{Cu}-\mathrm{K}_{\alpha 1}\right)$ for each material composition, and azimuthal integration of GIWAXS patterns in $\mathbf{q}_{x y}-\mathbf{q}_{z}$. (PDF)

\section{AUTHOR INFORMATION}

\section{Corresponding Author}

*E-mail: laura.herz@physics.ox.ac.uk.

\section{Present Address}

G.E.E.: Bagley Hall, Department of Chemistry, University of Washington, WA 98195, USA 


\section{Author Contributions}

The manuscript was written through contributions of all authors. All authors have given approval to the final version of the manuscript.

\section{Notes}

The authors declare no competing financial interest.

\section{ACKNOWLEDGMENTS}

The authors thank the Engineering and Physical Sciences Research Council (EPSRC) for financial support. We thank the Science and Technology Facilities Council (STFC) for support through the Challenge-Led Applied Systems Programme (ST/ L003309/1) and access to Diamond Light Source Beamline I07 (SI12690-1), and we acknowledge the assistance of Dr. Chris Nicklin in the facilitation of the GIWAXS experiments. R.J.S. is a Commonwealth Scholar funded by the UK government.

\section{REFERENCES}

(1) Green, M. A.; Ho-Baillie, A.; Snaith, H. J. Nat. Photonics 2014, 8, 506-514.

(2) Wang, B. H.; Xiao, X. D.; Chen, T. Nanoscale 2014, 6, 1228712297.

(3) Baikie, T.; Fang, Y. N.; Kadro, J. M.; Schreyer, M.; Wei, F. X.; Mhaisalkar, S. G.; Grätzel, M.; White, T. J. J. Mater. Chem. A 2013, 1, 5628.

(4) Liu, M. Z.; Johnston, M. B.; Snaith, H. J. Nature 2013, 501, 395.

(5) Johnston, M. B.; Herz, L. M. Acc. Chem. Res. 2016, 49, 146.

(6) Herz, L. M. Annu. Rev. Phys. Chem. 2016, 67, 65.

(7) Manser, J. S.; Christians, J. A.; Kamat, P. V. Chem. Rev. 2016, DOI: 10.1021 acs.chemrev.6b00136.

(8) Christians, J. A.; Miranda Herrera, P. A.; Kamat, P. V. J. Am. Chem. Soc. 2015, 137, 1530-1538.

(9) Wang, D.; Wright, M.; Elumalai, N. K.; Uddin, A. Sol. Energy Mater. Sol. Cells 2016, 147, 255.

(10) Niu, G. D.; Guo, X. D.; Wang, L. D. J. Mater. Chem. A 2015, 3, 8970

(11) Tiep, N. H.; Ku, Z. L.; Fan, H. J. Adv. Energy Mater. 2016, 6, 1501420.

(12) Clever, H. L.; Johnston, F. J. J. Phys. Chem. Ref. Data 1980, 9, 751.

(13) Patel, J. B.; Milot, R. L.; Wright, A. D.; Herz, L. M.; Johnston, M. B. J. Phys. Chem. Lett. 2016, 7, 96.

(14) Weerasinghe, H. C.; Dkhissi, Y.; Scully, A. D.; Caruso, R. A.; Cheng, Y. B. Nano Energy 2015, 18, 118.

(15) Zheng, F.; Saldana-Greco, D.; Liu, S.; Rappe, A. M. J. Phys. Chem. Lett. 2015, 6, 4862.

(16) Habisreutinger, S. N.; Leijtens, T.; Eperon, G. E.; Stranks, S. D.; Nicholas, R. J.; Snaith, H. J. Nano Lett. 2014, 14, 5561.

(17) Eperon, G. E.; Stranks, S. D.; Menelaou, C.; Johnston, M. B.; Herz, L. M.; Snaith, H. J. Energy Environ. Sci. 2014, 7, 982.

(18) Filip, M. R.; Eperon, G. E.; Snaith, H. J.; Giustino, F. Nat. Commun. 2014, 5, 5757.

(19) Mitzi, D. B.; Chondroudis, K.; Kagan, C. R. IBM J. Res. Dev. 2001, 45, 29.

(20) Tabuchi, Y.; Asai, K.; Rikukawa, M.; Sanui, K.; Ishigure, K. J. Phys. Chem. Solids 2000, 61, 837.

(21) Cao, D. H.; Stoumpos, C. C.; Farha, O. K.; Hupp, J. T.; Kanatzidis, M. G. J. Am. Chem. Soc. 2015, 137, 7843.

(22) Smith, I. C.; Hoke, E. T.; Solis-Ibarra, D.; McGehee, M. D.; Karunadasa, H. I. Angew. Chem., Int. Ed. 2014, 53, 11232.

(23) Ahmad, S.; Kanaujia, P. K.; Beeson, H. J.; Abate, A.; Deschler, F.; Credgington, D.; Steiner, U.; Prakash, G. V.; Baumberg, J. J. ACS Appl. Mater. Interfaces 2015, 7, 25227.

(24) Dou, L. T.; Wong, A. B.; Yu, Y.; Lai, M. L.; Kornienko, N.; Eaton, S. W.; Fu, A.; Bischak, C. G.; Ma, J.; Ding, T. N.; Ginsberg, N. S.; Wang, L. W.; Alivisatos, A. P.; Yang, P. D. Science 2015, 349, 1518.
(25) Tsai, H.; Nie, W.; Blancon, J.-C.; Stoumpos, C. C.; Asadpour, R.; Harutyunyan, B.; Neukirch, A. J.; Verduzco, R.; Crochet, J. J.; Tretiak, S.; Pedesseau, L.; Even, J.; Alam, M. A.; Gupta, G.; Lou, J.; Ajayan, P. M.; Bedzyk, M. J.; Kanatzidis, M. G.; Mohite, A. D. Nature 2016, 536, 312

(26) Koh, T. M.; Shanmugam, V.; Schlipf, J.; Oesinghaus, L.; MüllerBuschbaum, P.; Ramakrishnan, N.; Swamy, V.; Mathews, N.; Boix, P. P.; Mhaisalkar, S. G. Adv. Mater. 2016, 28, 3653.

(27) Byun, J.; Cho, H.; Wolf, C.; Jang, M.; Sadhanala, A.; Friend, R H.; Yang, H.; Lee, T.-W. Adv. Mater. 2016, 28, 7515.

(28) Hong, X.; Ishihara, T.; Nurmikko, A. V. Phys. Rev. B: Condens. Matter Mater. Phys. 1992, 45, 6961.

(29) Even, J.; Pedesseau, L.; Katan, C. ChemPhysChem 2014, 15, 3733.

(30) Sapori, D.; Kepenekian, M.; Pedesseau, L.; Katan, C.; Even, J. Nanoscale 2016, 8, 6369.

(31) Hong, X.; Ishihara, T.; Nurmikko, A. V. Solid State Commun. 1992, 84, 657.

(32) Stoumpos, C. C.; Cao, D. H.; Clark, D. J.; Young, J.; Rondinelli, J. M.; Jang, J. I.; Hupp, J. T.; Kanatzidis, M. G. Chem. Mater. 2016, 28, 2852.

(33) Ishihara, T. J. Lumin. 1994, 60, 269-274.

(34) Dammak, T.; Koubaa, M.; Boukheddaden, K.; Bougzhala, H.; Mlayah, A.; Abid, Y. J. Phys. Chem. C 2009, 113, 19305.

(35) Lanty, G.; Jemli, K.; Wei, Y.; Leymarie, J.; Even, J.; Lauret, J. S.; Deleporte, E. J. Phys. Chem. Lett. 2014, 5, 3958.

(36) Mikhnenko, O. V.; Blom, P. W. M.; Nguyen, T. Q. Energy Environ. Sci. 2015, 8, 1867.

(37) Chen, C. W.; Hsiao, S. Y.; Chen, C. Y.; Kang, H. W.; Huang, Z. Y.; Lin, H. W. J. Mater. Chem. A 2015, 3, 9152.

(38) D'Innocenzo, V.; Kandada, A. R. S.; De Bastiani, M.; Gandini, M.; Petrozza, A. J. Am. Chem. Soc. 2014, 136, 17730-17733.

(39) Grancini, G.; Marras, S.; Prato, M.; Giannini, C.; Quarti, C.; De Angelis, F.; De Bastiani, M.; Eperon, G. E.; Snaith, H. J.; Manna, L.; Petrozza, A. J. Phys. Chem. Lett. 2014, 5, 3836.

(40) Dammak, T.; Fourati, N.; Boughzala, H.; Mlayah, A.; Abid, Y. J. Lumin. 2007, 127, 404.

(41) Fox, M. Optical Properties of Solids; 2nd ed.; Oxford University Press: Oxford, UK, 2001.

(42) Milot, R. L.; Eperon, G. E.; Snaith, H. J.; Johnston, M. B.; Herz, L. M. Adv. Funct. Mater. 2015, 25, 6218.

(43) Noel, N. K.; Abate, A.; Stranks, S. D.; Parrott, E. S.; Burlakov, V. M.; Goriely, A.; Snaith, H. J. ACS Nano 2014, 8, 9815.

(44) Parrott, E. S.; Milot, R. L.; Stergiopoulos, T.; Snaith, H. J.; Johnston, M. B.; Herz, L. M. J. Phys. Chem. Lett. 2016, 7, 1321.

(45) Yao, K.; Wang, X. F.; Li, F.; Zhou, L. Chem. Commun. 2015, 51, 15430.

(46) Yuan, M.; Quan, L. N.; Comin, R.; Walters, G.; Sabatini, R.; Voznyy, O.; Hoogland, S.; Zhao, Y.; Beauregard, E. M.; Kanjanaboos, P.; Lu, Z.; Kim, D. H.; Sargent, E. H. Nat. Nano 2016, advance online publication; DOI:10.1038/nnano.2016.110.

(47) Haug, A. J. Phys. C: Solid State Phys. 1983, 16, 4159.

(48) Lochmann, W. Phys. Status Solidi A-Appl. Res. 1977, 40, 285.

(49) Takeshima, M. Phys. Rev. B: Condens. Matter Mater. Phys. 1982, 25, 5390 .

(50) Polkovnikov, A. S.; Zegrya, G. G. Phys. Rev. B: Condens. Matter Mater. Phys. 1998, 58, 4039.

(51) Klimov, V. I.; Mikhailovsky, A. A.; McBranch, D. W.; Leatherdale, C. A.; Bawendi, M. G. Science 2000, 287, 1011-1013.

(52) Robel, I.; Gresback, R.; Kortshagen, U.; Schaller, R. D.; Klimov, V. I. Phys. Rev. Lett. 2009, 102, 177404.

(53) Bertazzi, F.; Zhou, X. Y.; Goano, M.; Ghione, G.; Bellotti, E. Appl. Phys. Lett. 2013, 103, 081106.

(54) Quan, L. N.; Yuan, M. J.; Comin, R.; Voznyy, O.; Beauregard, E. M.; Hoogland, S.; Buin, A.; Kirmani, A. R.; Zhao, K.; Amassian, A.; Kim, D. H.; Sargent, E. H. J. Am. Chem. Soc. 2016, 138, 2649. 